\title{
Transition State Stabilization and $\alpha$-Amino Carbon Acidity in Alanine Racemase
}

\author{
Dan T. Major, ${ }^{\star}$ Kwangho Nam, Jiali Gao* \\ Department of Chemistry and Supercomputing Institute, Digital Technology Center, University of \\ Minnesota, Minneapolis, Minnesota 55455
}

Tables and Figures containing information on the structures, energies, and electronic properties of molecules that have been used in the parameterization of the reaction specific parameters (SRP) for the semiempirical Austin model 1 (AM1) formulism. The target data include experimental heats of formation and dipole moments, and energies of reaction, partial charges and geometries from density functional theory (DFT) calculations using the mPW1PW91/6-311++G(3df,2p)//mPW1PW91/6-31+G(d) model. The performance of the SRP-AM1 model is also compared with results obtained from the DFT calculations. A total of 4 tables and 18 pages are included in this supplementary material.

Table S1. SRP-AM1 parameters ${ }^{a}$ for the AlaRM and model deprotonation of PLP-Ala by phenoxide reactions and the model deprotonation reaction of Ala by phenoxide.

\begin{tabular}{lrrrrrr} 
& $\mathrm{C}$ & \multicolumn{5}{c}{$\mathrm{N}$} \\
\cline { 2 - 7 } & AM1 & $\begin{array}{r}\text { AM1-SRP } \\
\text { (AlaRM) }\end{array}$ & $\begin{array}{r}\text { AM1-SRP } \\
\text { (Ala) }\end{array}$ & AM1 & $\begin{array}{r}\text { AM1-SRP } \\
\text { (AlaRM) }\end{array}$ & $\begin{array}{r}\text { AM1-SRP } \\
\text { (Ala) }\end{array}$ \\
\hline $\mathrm{U}_{\mathrm{ss}}$ & -52.028658 & -52.220373 & -52.053310 & -71.860000 & -71.443604 & -72.525445 \\
$\mathrm{U}_{\mathrm{pp}}$ & -39.614239 & -39.449134 & -38.937879 & -57.167581 & -57.085525 & -58.295047 \\
$\mathrm{G}_{\mathrm{ss}}$ & 12.230000 & 12.200604 & 12.220621 & 13.590000 & 13.589907 & 13.750393 \\
$\mathrm{G}_{\mathrm{sp}}$ & 11.470000 & 11.702499 & 11.547749 & 12.660000 & 12.695848 & 12.347239 \\
$\mathrm{G}_{\mathrm{pp}}$ & 11.080000 & 11.073049 & 10.989099 & 12.980000 & 13.106802 & 13.069911 \\
$\mathrm{G}_{\mathrm{p} 2}$ & 9.840000 & 9.840000 & 9.845988 & 11.590000 & 11.557655 & 11.543123 \\
$\mathrm{H}_{\mathrm{sp}}$ & 2.430000 & 2.432422 & 2.408146 & 3.140000 & 3.140011 & 3.150527 \\
$\alpha$ & 2.648274 & 2.648791 & 2.663961 & 2.947286 & 2.947286 & 2.941656 \\
$\beta_{\mathrm{s}}$ & -15.715783 & -15.652257 & -16.023842 & -20.299110 & -20.396854 & -20.142877 \\
$\beta_{\mathrm{p}}$ & -7.719283 & -7.806939 & -7.763327 & -18.238666 & -18.294382 & -17.761336 \\
$\zeta_{\mathrm{s}}$ & 1.808665 & 1.813455 & 1.808298 & 2.315410 & 2.307563 & 2.386050 \\
$\zeta_{\mathrm{p}}$ & 1.685116 & 1.683855 & 1.684205 & 2.157940 & 2.167921 & 2.211587 \\
$\mathrm{FN} 11$ & 0.011355 & 0.011355 & 0.011469 & 0.025251 & 0.035504 & 0.029741 \\
FN21 & 5.000000 & 5.000000 & 4.987789 & 5.000000 & 5.000000 & 5.020552 \\
FN31 & 1.600000 & 1.600000 & 1.589515 & 1.500000 & 1.500070 & 1.487454 \\
FN12 & 0.045924 & 0.045924 & 0.045404 & 0.028953 & 0.033803 & 0.037028 \\
FN22 & 5.000000 & 5.000000 & 4.977350 & 5.000000 & 4.999513 & 5.017092 \\
FN32 & 1.850000 & 1.850000 & 1.842081 & 2.100000 & 2.126830 & 2.142630 \\
FN13 & -0.020061 & -0.025161 & -0.019202 & -0.005806 & 0.004252 & -0.004421 \\
FN23 & 5.000000 & 5.000000 & 5.033816 & 2.000000 & 2.000000 & 2.006230 \\
FN33 & 2.050000 & 2.050000 & 2.021158 & 2.400000 & 2.400000 & 2.386031 \\
FN14 & -0.001260 & -0.001260 & -0.001190 & & & \\
FN24 & 5.000000 & 5.003199 & 5.016608 & & & \\
FN34 & 2.650000 & 2.650002 & 2.670871 & & & \\
\hline & & & & & &
\end{tabular}


Table S1. Continued

\begin{tabular}{|c|c|c|c|c|c|c|}
\hline & \multicolumn{3}{|l|}{$\mathrm{O}$} & \multicolumn{3}{|l|}{$\mathrm{H}$} \\
\hline & & AM1-SRP & AM1-SRP & & AM1-SRP & AM1-SRP \\
\hline & AMI & (AlaRM) & (Ala) & AMI & (AlaRM) & (Ala) \\
\hline $\mathrm{U}_{\mathrm{ss}}$ & -97.830000 & -98.784021 & -97.667460 & -11.396427 & -11.189614 & -11.136918 \\
\hline $\mathrm{U}_{\mathrm{pp}}$ & -78.262380 & -78.278872 & -78.237630 & & & \\
\hline $\mathrm{G}_{\mathrm{ss}}$ & 15.420000 & 15.420000 & 15.580126 & 12.848000 & 13.187186 & 13.187628 \\
\hline $\mathrm{G}_{\mathrm{sp}}$ & 14.480000 & 14.338469 & 14.382241 & & & \\
\hline $\mathrm{G}_{\mathrm{pp}}$ & 14.520000 & 14.519992 & 14.753680 & & & \\
\hline $\mathrm{G}_{\mathrm{p} 2}$ & 12.980000 & 12.953517 & 12.894272 & & & \\
\hline $\mathrm{H}_{\mathrm{sp}}$ & 3.940000 & 3.940000 & 4.039532 & & & \\
\hline$\alpha$ & 4.455371 & 4.455283 & 4.509602 & 2.882324 & 2.914021 & 2.869398 \\
\hline$\beta_{\mathrm{s}}$ & -29.272773 & -28.675427 & -28.830671 & -6.173787 & -6.281752 & -6.396574 \\
\hline$\beta_{\mathrm{p}}$ & -29.272773 & -29.272773 & -28.952650 & & & \\
\hline$\zeta_{\mathrm{s}}$ & 3.108032 & 3.126217 & 3.118294 & 1.188078 & 1.198675 & 1.184705 \\
\hline$\zeta_{\mathrm{p}}$ & 2.524039 & 2.536238 & 2.552184 & & & \\
\hline FN11 & 0.280962 & 0.283401 & 0.255866 & 0.122796 & 0.122796 & 0.120812 \\
\hline FN21 & 5.000000 & 5.000089 & 4.910492 & 5.000000 & 5.000050 & 5.090316 \\
\hline FN31 & 0.847918 & 0.839512 & 0.822207 & 1.200000 & 1.233031 & 1.233165 \\
\hline FN12 & 0.081430 & 0.081434 & 0.086837 & 0.005090 & 0.005090 & 0.003658 \\
\hline FN22 & 7.000000 & 6.998392 & 7.066003 & 5.000000 & 5.000000 & 4.967418 \\
\hline FN32 & 1.445071 & 1.457284 & 1.444917 & 1.800000 & 1.800000 & 1.760482 \\
\hline FN13 & & & & -0.018336 & -0.018336 & -0.018336 \\
\hline $\mathrm{FN} 23$ & & & & 2.000000 & 2.029834 & 2.036884 \\
\hline FN33 & & & & 2.100000 & 2.090036 & 2.102692 \\
\hline FN14 & & & & & & \\
\hline FN24 & & & & & & \\
\hline FN34 & & & & & & \\
\hline
\end{tabular}

${ }^{a}$ Timothy J. Giese and Darrin M. York, "Nonlinear parameter optimization of semiempirical quantum methods for potential energy surfaces", in preparation. 
Table S2. Molecular descriptors ${ }^{a}$ used in AM1 parameter optimization obtained with SRP-AM1 and ab-initio methods or experiment for the AlaRM reaction and a model deprotonation reaction of PLP-Ala by phenoxide.

\begin{tabular}{rrll}
$\mathrm{C}_{6} \mathrm{H}_{5} \mathrm{O}^{-}$ & & & \\
SRP-AM1 & Exp/ab-initio & Property & Source \\
\hline-42.81 & -39.07 & Heat of formation & Exp $^{c}$ \\
1.3951 & 1.4023 & C-C Bond & mPW1PW91 $^{b}$ \\
1.3950 & 1.4023 & C-C Bond & mPW1PW91 \\
1.3757 & 1.3876 & C-C Bond & mPW1PW91 \\
1.4334 & 1.4435 & C-C Bond & mPW1PW91 \\
1.4330 & 1.4435 & C-C Bond & mPW1PW91 \\
1.0912 & 1.0915 & C-H Bond & mPW1PW91 \\
1.0844 & 1.0874 & C-H Bond & mPW1PW91 \\
1.0911 & 1.0915 & C-H Bond & mPW1PW91 \\
1.0862 & 1.0889 & C-H Bond & mPW1PW91 \\
1.0861 & 1.0889 & C-H Bond & mPW1PW91 \\
1.2785 & 1.2690 & C-O Bond & mPW1PW91 \\
118.65 & 117.45 & H-C-H Angle & mPW1PW91 \\
121.32 & 121.71 & H-C-H Angle & mPW1PW91 \\
121.24 & 122.66 & H-C-H Angle & mPW1PW91 \\
116.22 & 113.79 & H-C-C Angle & mPW1PW91 \\
121.12 & 122.66 & H-C-C Angle & mPW1PW91 \\
121.45 & 121.71 & H-C-C Angle & mPW1PW91 \\
121.88 & 123.10 & C-C-O Angle & mPW1PW91 \\
121.90 & 123.10 & C-C-O Angle & mPW1PW91
\end{tabular}

Table S2. Continued

\begin{tabular}{rrll}
\hline $\mathrm{C}_{6} \mathrm{H}_{5} \mathrm{OH}$ & & & \\
SRP-AM1 & Exp/ab-initio & Property & Source $^{\mathrm{b}, \mathrm{c}}$ \\
\hline-27.71 & -23.04 & Heat of formation & Exp $^{c}$ \\
350.60 & 350.5 & Proton Affinity & Exp $^{c}$ \\
1.3933 & 1.3953 & C-C Bond & mPW1PW91 $^{b}$ \\
1.3908 & 1.3922 & C-C Bond & mPW1PW91 \\
1.3913 & 1.3936 & C-C Bond & mPW1PW91 \\
1.3953 & 1.3952 & C-C Bond & mPW1PW91 \\
1.3984 & 1.3954 & C-C Bond & mPW1PW91 \\
1.0905 & 1.0858 & C-H Bond & mPW1PW91 \\
1.0894 & 1.0849 & C-H Bond & mPW1PW91 \\
1.0904 & 1.0858 & C-H Bond & mPW1PW91 \\
1.0880 & 1.0878 & C-H Bond & mPW1PW91
\end{tabular}




$\begin{array}{llll}1.0886 & 1.0847 & \text { C-H Bond } & \text { mPW1PW91 } \\ 1.3828 & 1.3628 & \text { C-O Bond } & \text { mPW1PW91 } \\ 0.9702 & 0.9657 & \text { O-H Bond } & \text { mPW1PW91 } \\ 120.20 & 119.23 & \text { H-C-H Angle } & \text { mPW1PW91 } \\ 120.36 & 120.56 & \text { H-C-H Angle } & \text { mPW1PW91 } \\ 118.67 & 119.73 & \text { H-C-H Angle } & \text { mPW1PW91 } \\ 121.75 & 120.12 & \text { H-C-C Angle } & \text { mPW1PW91 } \\ 118.46 & 119.59 & \text { H-C-C Angle } & \text { mPW1PW91 } \\ 120.56 & 120.77 & \text { H-C-C Angle } & \text { mPW1PW91 } \\ 122.20 & 122.61 & \text { C-C-O Angle } & \text { mPW1PW91 } \\ 116.04 & 117.27 & \text { C-C-O Angle } & \text { mPW1PW91 } \\ 1.4535 & 1.2886 & \text { Dipole moment } & \text { mPW1PW91 } \\ 3523.2 & 3732.5 & \text { O-H frequency } & \text { mPW1PW9 }{ }^{b}\end{array}$

Table S2. Continued

\begin{tabular}{rrll}
\hline $\mathrm{C}_{2} \mathrm{H}_{5} \mathrm{NH}_{2}$ & & & \\
SRP-AM1 & Exp/ab-initio & Prop. & Source \\
\hline-20.60 & -11.35 & Heat of formation & Exp $^{c}$ \\
1.5305 & 1.5277 & C-C Bond & mPW1PW91 $^{b}$ \\
1.4294 & 1.4581 & C-N Bond & mPW1PW91 $^{b}$ \\
1.1061 & 1.0961 & C-H Bond & mPW1PW91 $^{b}$ \\
1.1063 & 1.0961 & C-H Bond & mPW1PW91 $^{b}$ \\
1.1064 & 1.0963 & C-H Bond & mPW1PW91 $^{b}$ \\
1.1172 & 1.0958 & C-H Bond & mPW1PW91 $^{b}$ \\
1.1172 & 1.0958 & C-H Bond & mPW1PW91 \\
0.9798 & 1.0157 & N-H Bond & mPW1PW91 \\
0.9794 & 1.0157 & N-H Bond & mPW1PW91 $^{b}$ \\
117.23 & 116.04 & C-C-N Angle & mPW1PW91 \\
95.76 & 95.62 & C-N-H Angle & mPW1PW91 $^{b}$ \\
144.70 & 140.97 & C-N-H Angle & mPW1PW91 $^{b}$ \\
112.60 & 106.77 & H-N-H Angle & mPW1PW91 $^{b}$ \\
1.3554 & 1.2903 & Dipole moment & mPW1PW91 $^{b}$ \\
3657.6 & 3615.9 & N-H frequency & mPW1PW91 $^{b}$ \\
3628.7 & 3511.7 & N-H frequency & mPW1PW91 $^{b}$
\end{tabular}


Table S2. Continued

\begin{tabular}{|c|c|c|c|}
\hline $\begin{array}{l}\mathrm{C}_{2} \mathrm{H}_{5} \mathrm{NH}_{3}{ }^{+} \\
\text {SRP-AM1 }\end{array}$ & Exp/ab-initio & Prop. & Source \\
\hline 124.58 & 136.38 & Heat of formation & $\operatorname{Exp}^{c}$ \\
\hline 220.52 & 219.9 & Proton Affinity & $\operatorname{Exp}^{c}$ \\
\hline 1.5180 & 1.5122 & C-C Bond & mPW1PW91 ${ }^{b}$ \\
\hline 1.5036 & 1.5165 & C-N Bond & mPW1PW91 ${ }^{b}$ \\
\hline 1.1076 & 1.0940 & C-H Bond & mPW1PW91 $^{b}$ \\
\hline 1.1077 & 1.0940 & C-H Bond & mPW1PW91 ${ }^{b}$ \\
\hline 1.1118 & 1.0926 & $\mathrm{C}-\mathrm{H}$ Bond & mPW1PW91 $^{b}$ \\
\hline 1.1176 & 1.0913 & C-H Bond & mPW1PW91 ${ }^{b}$ \\
\hline 1.1176 & 1.0913 & C-H Bond & mPW1PW91 ${ }^{b}$ \\
\hline 1.0118 & 1.0248 & N-H Bond & mPW1PW91 $^{b}$ \\
\hline 1.0114 & 1.0248 & N-H Bond & mPW1PW91 ${ }^{b}$ \\
\hline 1.0108 & 1.0246 & N-H Bond & mPW1PW91 ${ }^{b}$ \\
\hline 113.38 & 110.52 & C-C-N Angle & mPW1PW91 $^{b}$ \\
\hline 93.81 & 95.71 & C-N-H Angle & mPW1PW91 ${ }^{b}$ \\
\hline 140.18 & 141.17 & C-N-H Angle & mPW1PW91 $^{b}$ \\
\hline 92.58 & 94.56 & C-N-H Angle & mPW1PW91 $^{b}$ \\
\hline 108.72 & 107.00 & H-N-H Angle & mPW1PW91 $^{b}$ \\
\hline 109.32 & 107.51 & H-N-H Angle & mPW1PW91 ${ }^{b}$ \\
\hline 109.32 & 107.51 & H-N-H Angle & mPW1PW91 ${ }^{b}$ \\
\hline 3479.9 & 3521.0 & $\mathrm{~N}-\mathrm{H}$ frequency & mPW1PW91 ${ }^{b}$ \\
\hline 3358.2 & 3519.4 & $\mathrm{~N}-\mathrm{H}$ frequency & mPW1PW91 $^{b}$ \\
\hline 3351.1 & 3410.9 & $\mathrm{~N}-\mathrm{H}$ frequency & mPW1PW91 ${ }^{b}$ \\
\hline
\end{tabular}

Table S2. Continued

\begin{tabular}{rrll}
\hline \multicolumn{1}{l}{$\begin{array}{l}\text { PLP-Ala } \\
\text { RS-AM1 }\end{array}$} & Exp/ab-initio & Prop. & \\
\hline-50.34 & ND & Heat of formation & \\
1.3917 & 1.4080 & C-C Bond & mPW1PW91 $^{b}$ \\
1.4079 & 1.3936 & C-C Bond & mPW1PW91 $^{b}$ \\
1.3400 & 1.3405 & C-N Bond & mPW1PW91 $^{b}$ \\
1.3292 & 1.3418 & N-C Bond & mPW1PW91 \\
1.4480 & 1.4290 & C-C Bond & mPW1PW91 \\
1.4434 & 1.4557 & C-C Bond & mPW1PW91 \\
1.0880 & 1.0926 & C-H Bond & mPW1PW91 \\
1.0893 & 1.0921 & C-H Bond & mPW1PW91 \\
1.0903 & 1.0918 & C-H Bond & mPW1PW91
\end{tabular}




\begin{tabular}{|c|c|c|c|}
\hline 1.2760 & 1.2771 & C-O Bond & mPW1PW91 ${ }^{b}$ \\
\hline 1.4419 & 1.4264 & C-C Bond & $\mathrm{mPW} 1 \mathrm{PW} 91^{b}$ \\
\hline 1.3377 & 1.3516 & C-N Bond & mPW1PW91 ${ }^{b}$ \\
\hline 1.3429 & 1.3332 & N-C Bond & mPW1PW91 ${ }^{b}$ \\
\hline 1.4863 & 1.4834 & C-C Bond & mPW1PW91 $^{b}$ \\
\hline 1.5053 & 1.5048 & C-C Bond & mPW1PW91 $^{b}$ \\
\hline 1.2760 & 1.2692 & C-O Bond & mPW1PW91 $^{b}$ \\
\hline 1.2719 & 1.2582 & C-O Bond & mPW1PW91 $^{b}$ \\
\hline 1.0929 & 1.0856 & C-H Bond & mPW1PW91 $^{b}$ \\
\hline 1.0022 & 1.0382 & N-H Bond & mPW1PW91 $^{b}$ \\
\hline 1.1121 & 1.1073 & C-H Bond & mPW1PW91 $^{b}$ \\
\hline 1.1089 & 1.0917 & C-H Bond & mPW1PW91 ${ }^{b}$ \\
\hline 1.1120 & 1.1073 & C-H Bond & mPW1PW91 $^{b}$ \\
\hline 120.75 & 121.56 & C-C-C Angle & mPW1PW91 $^{b}$ \\
\hline 123.17 & 123.01 & C-C-N Angle & mPW1PW91 ${ }^{b}$ \\
\hline 117.09 & 116.42 & C-N-C Angle & $\mathrm{mPW} 1 \mathrm{PW} 91^{b}$ \\
\hline 125.74 & 127.10 & N-C-C Angle & mPW1PW91 ${ }^{b}$ \\
\hline 115.45 & 114.89 & C-C-C Angle & mPW1PW91 ${ }^{b}$ \\
\hline 117.01 & 120.55 & C-C-C Angle & mPW1PW91 $^{b}$ \\
\hline 125.19 & 122.42 & C-C-C Angle & mPW1PW91 $^{b}$ \\
\hline 124.14 & 121.96 & C-C-O Angle & $\mathrm{mPW} 1 \mathrm{PW} 91^{b}$ \\
\hline 120.41 & 123.15 & C-C-O Angle & mPW1PW91 ${ }^{b}$ \\
\hline 127.66 & 124.86 & C-C-N Angle & mPW1PW91 ${ }^{b}$ \\
\hline 124.50 & 128.07 & C-N-C Angle & mPW1PW91 ${ }^{b}$ \\
\hline 121.75 & 118.23 & N-C-C Angle & mPW1PW91 ${ }^{b}$ \\
\hline 122.33 & 119.84 & N-C-C Angle & mPW1PW91 ${ }^{b}$ \\
\hline 117.71 & 115.53 & C-C-O Angle & $\mathrm{mPW}$ PW91 ${ }^{b}$ \\
\hline 120.62 & 116.65 & C-C-O Angle & mPW1PW91 ${ }^{b}$ \\
\hline 118.18 & 114.26 & C-N-H Angle & $\mathrm{mPW} 1 \mathrm{PW} 91^{b}$ \\
\hline 117.31 & 117.67 & C-N-H Angle & mPW1PW91 ${ }^{b}$ \\
\hline
\end{tabular}


Table S2. Continued

\begin{tabular}{|c|c|c|c|}
\hline $\begin{array}{l}\text { PLP-Ala } \\
\text { RS-AM1 }\end{array}$ & Exp/ab-initio & Prop. & Source \\
\hline-101.69 & ND & Heat of formation & \\
\hline 417.05 & 417.7 & Proton Affinity & mPW1PW91 ${ }^{b}$ \\
\hline 1.4260 & 1.4167 & C-C Bond & mPW1PW91 ${ }^{b}$ \\
\hline 1.3724 & 1.3708 & C-C Bond & mPW1PW91 ${ }^{b}$ \\
\hline 1.3768 & 1.3648 & C-N Bond & mPW1PW91 ${ }^{b}$ \\
\hline 1.2977 & 1.3067 & $\mathrm{~N}-\mathrm{C}$ Bond & mPW1PW91 ${ }^{b}$ \\
\hline 1.4857 & 1.4597 & C-C Bond & mPW1PW91 ${ }^{b}$ \\
\hline 1.4374 & 1.4487 & C-C Bond & mPW1PW91 ${ }^{b}$ \\
\hline 1.0896 & 1.0895 & C-H Bond & mPW1PW91 ${ }^{b}$ \\
\hline 1.0907 & 1.0862 & C-H Bond & mPW1PW91 ${ }^{b}$ \\
\hline 1.0947 & 1.0897 & $\mathrm{C}-\mathrm{H}$ Bond & mPW1PW91 ${ }^{b}$ \\
\hline 1.2574 & 1.2495 & C-O Bond & mPW1PW91 ${ }^{b}$ \\
\hline 1.4102 & 1.4137 & C-C Bond & mPW1PW91 ${ }^{b}$ \\
\hline 1.3196 & 1.3032 & C-N Bond & mPW1PW91 ${ }^{b}$ \\
\hline 1.4553 & 1.4609 & N-C Bond & mPW1PW91 ${ }^{b}$ \\
\hline 1.5298 & 1.5184 & C-C Bond & mPW1PW91 ${ }^{b}$ \\
\hline 1.5749 & 1.5794 & C-C Bond & mPW1PW91 ${ }^{b}$ \\
\hline 1.2616 & 1.2460 & C-O Bond & mPW1PW91 ${ }^{b}$ \\
\hline 1.2594 & 1.2481 & C-O Bond & mPW1PW91 ${ }^{b}$ \\
\hline 1.1062 & 1.0892 & $\mathrm{C}-\mathrm{H}$ Bond & mPW1PW91 ${ }^{b}$ \\
\hline 0.9946 & 1.0337 & N-H Bond & mPW1PW91 ${ }^{b}$ \\
\hline 1.1065 & 1.0974 & C-H Bond & mPW1PW91 ${ }^{b}$ \\
\hline 1.1080 & 1.0916 & C-H Bond & mPW1PW91 ${ }^{b}$ \\
\hline 1.1070 & 1.0957 & C-H Bond & mPW1PW91 ${ }^{b}$ \\
\hline 1.1170 & 1.0995 & C-H Bond L-Ala & mPW1PW91 ${ }^{b}$ \\
\hline 120.63 & 120.45 & C-C-C Angle & mPW1PW91 ${ }^{b}$ \\
\hline 123.27 & 122.43 & C-C-N Angle & mPW1PW91 ${ }^{b}$ \\
\hline 117.88 & 118.62 & C-N-C Angle & mPW1PW91 ${ }^{b}$ \\
\hline 125.20 & 125.73 & N-C-C Angle & mPW1PW91 ${ }^{b}$ \\
\hline 115.16 & 113.87 & C-C-C Angle & mPW1PW91 ${ }^{b}$ \\
\hline 117.69 & 119.58 & C-C-C Angle & mPW1PW91 ${ }^{b}$ \\
\hline 124.47 & 121.51 & C-C-C Angle & mPW1PW91 ${ }^{b}$ \\
\hline 125.13 & 123.89 & C-C-O Angle & mPW1PW91 ${ }^{b}$ \\
\hline 119.71 & 122.24 & C-C-O Angle & mPW1PW91 ${ }^{b}$ \\
\hline 128.75 & 126.86 & C-C-N Angle & mPW1PW91 ${ }^{b}$ \\
\hline 121.80 & 125.78 & C-N-C Angle & mPW1PW91 ${ }^{b}$ \\
\hline 113.70 & 113.75 & N-C-C Angle & mPW1PW91 ${ }^{b}$ \\
\hline 113.57 & 108.37 & N-C-C Angle & mPW1PW91 $1^{b}$ \\
\hline
\end{tabular}




$\begin{array}{llll}114.47 & 114.12 & \text { C-C-O Angle } & \text { mPW1PW91 } \\ 118.38 & 114.47 & \text { C-C-O Angle } & \text { mPW1PW91 } \\ 121.90 & 119.88 & \text { C-N-H Angle } & \text { mPW1PW91 } \\ 116.29 & 114.25 & \text { C-N-H Angle } & \text { mPW1PW91 } \\ 107.95 & 107.29 & \text { N-C-H Angle } & \text { mPW1PW91 } \\ 108.62 & 109.27 & \text { C-C-H Angle } & \text { mPW1PW91 } \\ 107.25 & 104.77 & \text { C-C-H Angle } & \text { mPW1PW91 } \\ 3467.2 & 3682.6 & \text { Frequency } & \text { mPW1PW91 } \\ 3277.7 & 3386.1 & \text { Frequency } & \text { mPW1PW91 } \\ 3261.4 & 3373.1 & \text { Frequency } & \text { mPW1PW91 } \\ 3232.7 & 3345.0 & \text { Frequency } & \text { mPW1PW91 } \\ 3231.2 & 3331.1 & \text { Frequency } & \text { mPW1PW91 } \\ 3151.4 & 3326.5 & \text { Frequency } & \text { mPW1PW91 } \\ 3145.3 & 3238.5 & \text { Frequency } & \text { mPW1PW91 } \\ 3141.5 & 3197.3 & \text { Frequency } & \text { mPW1PW91 }^{b} \\ 3086.3 & 3183.6 & \text { Frequency } & \text { mPW1PW91 }^{b}\end{array}$

${ }^{a}$ Energies are in units of $\mathrm{kcal} / \mathrm{mol}$, distances in $\AA$, angles in degrees, dipole moments in Debye, atomic charges in a.u., and frequencies in $\mathrm{cm}^{-1} .{ }^{b} \mathrm{mPW} 1 \mathrm{PW} 91 / 6-$

311++G(3df,2p)//mPW1PW91/6-31+G(d) ${ }^{c}$ Hunter, E.P.; Lias, S.G. J. Phys. Chem. Ref. Data 1998, 27, 413-656. 
Table S3. Molecular descriptors ${ }^{a}$ used in AM1 parameter optimization obtained with SRP-AM1 and ab-initio methods or experiment for the model deprotonation reaction of Ala by phenoxide.

\begin{tabular}{rrll}
\hline $\mathrm{C}_{6} \mathrm{H}_{5} \mathrm{O}^{-}$ & & & \\
SRP-AM1 & Exp/ab-initio & Property & Source \\
\hline-38.53 & -39.07 & Heat of formation & Exp $^{c}$ \\
1.3964 & 1.4023 & C-C Bond & mPW1PW91 $^{b}$ \\
1.3964 & 1.4023 & C-C Bond & mPW1PW91 \\
1.3767 & 1.3876 & C-C Bond & mPW1PW91 \\
1.4379 & 1.4435 & C-C Bond & mPW1PW91 \\
1.4377 & 1.4435 & C-C Bond & mPW1PW91 \\
1.0935 & 1.0915 & C-H Bond & mPW1PW91 \\
1.0867 & 1.0874 & C-H Bond & mPW1PW91 \\
1.0936 & 1.0915 & C-H Bond & mPW1PW91 \\
1.0879 & 1.0889 & C-H Bond & mPW1PW91 \\
1.0882 & 1.0889 & C-H Bond & mPW1PW91 \\
1.2582 & 1.2690 & C-O Bond & mPW1PW91 \\
118.69 & 117.45 & H-C-H Angle & mPW1PW91 \\
121.38 & 121.71 & H-C-H Angle & mPW1PW91 \\
121.26 & 122.66 & H-C-H Angle & mPW1PW91 \\
116.03 & 113.79 & H-C-C Angle & mPW1PW91 \\
121.19 & 122.66 & H-C-C Angle & mPW1PW91 \\
121.45 & 121.71 & H-C-C Angle & mPW1PW91 \\
121.98 & 123.10 & C-C-O Angle & mPW1PW91 \\
121.99 & 123.10 & C-C-O Angle & mPW1PW91
\end{tabular}

Table S3. Continued

\begin{tabular}{crll}
\hline $\mathrm{C}_{6} \mathrm{H}_{5} \mathrm{OH}$ & & & \\
SRP-AM1 & Exp/ab-initio & Property & Source \\
\hline-22.44 & -23.04 & Heat of formation & Exp $^{c}$ \\
349.61 & 350.5 & Proton Affinity & mPW1PW91 \\
1.3944 & 1.3953 & C-C Bond & mPW1PW91 \\
1.3918 & 1.3922 & C-C Bond & mPW1PW91 \\
1.3917 & 1.3936 & C-C Bond & mPW1PW91 \\
1.3996 & 1.3952 & C-C Bond & mPW1PW91 \\
1.4028 & 1.3954 & C-C Bond & mPW1PW91 \\
1.0915 & 1.0858 & C-H Bond & mPW1PW91 \\
1.0904 & 1.0849 & C-H Bond & mPW1PW91 \\
1.0913 & 1.0858 & C-H Bond & mPW1PW91 \\
1.0896 & 1.0878 & C-H Bond & mPW1PW91
\end{tabular}




$\begin{array}{llll}1.3604 & 1.3628 & \text { C-H Bond } & \text { mPW1PW91 } \\ 1.0892 & 1.0847 & \text { C-O Bond } & \text { mPW1PW91 } \\ 0.9685 & 0.9657 & \text { O-H Bond } & \text { mPW1PW91 } \\ 120.13 & 119.23 & \text { H-C-H Angle } & \text { mPW1PW91 } \\ 120.44 & 120.56 & \text { H-C-H Angle } & \text { mPW1PW91 } \\ 118.74 & 119.73 & \text { H-C-H Angle } & \text { mPW1PW91 } \\ 121.54 & 120.12 & \text { H-C-C Angle } & \text { mPW1PW91 } \\ 118.44 & 119.59 & \text { H-C-C Angle } & \text { mPW1PW91 } \\ 120.72 & 120.77 & \text { H-C-C Angle } & \text { mPW1PW91 } \\ 122.09 & 122.61 & \text { C-C-O Angle } & \text { mPW1PW91 } \\ 116.37 & 117.27 & \text { C-C-O Angle } & \text { mPW1PW91 } \\ 110.55 & 109.70 & \text { C-O-H Angle } & \text { mPW1PW91 }^{b} \\ 1.5439 & 1.2886 & \text { Dipole moment } & \text { mPW1PW91 }^{b} \\ 3615.5 & 3732.5 & \text { O-H frequency } & \text { mPW1PW91 }^{b}\end{array}$

Table S3. Continued

\begin{tabular}{|c|c|c|c|}
\hline $\begin{array}{l}\text { Ala }^{-} \\
\text {SRP-AM1 }\end{array}$ & Exp/ab-initio & Prop. & Source \\
\hline-94.89 & ND & $\begin{array}{l}\text { Heat of formation } \\
\text { Heat of formation }\end{array}$ & \\
\hline-26.39 & ND & Zwitter-ion & \\
\hline 1.4454 & 1.4496 & C-N Bond & $\mathrm{mPW}$ PW91 ${ }^{b}$ \\
\hline 1.4725 & 1.4894 & C-C Bond & mPW1PW91 $^{b}$ \\
\hline 1.3958 & 1.3944 & C-C Bond & mPW1PW91 $^{b}$ \\
\hline 1.3818 & 1.4065 & C-O Bond & mPW1PW91 $^{b}$ \\
\hline 1.2473 & 1.2502 & C-O Bond & mPW1PW91 ${ }^{b}$ \\
\hline 0.9626 & 0.9775 & O-H Bond & mPW1PW91 ${ }^{b}$ \\
\hline 0.9971 & 1.0212 & N-H Bond & $\mathrm{mPW} 1 \mathrm{PW} 91^{b}$ \\
\hline 0.9971 & 1.0212 & N-H Bond & mPW1PW91 ${ }^{b}$ \\
\hline 1.1171 & 1.1110 & C-H Bond & mPW1PW91 ${ }^{b}$ \\
\hline 1.1105 & 1.0953 & C-H Bond & mPW1PW91 $^{b}$ \\
\hline 1.1174 & 1.1109 & C-H Bond & mPW1PW91 ${ }^{b}$ \\
\hline 118.25 & 119.13 & N-C-C Angle & mPW1PW91 ${ }^{b}$ \\
\hline 121.29 & 118.13 & N-C-C Angle & mPW1PW91 $^{b}$ \\
\hline 120.45 & 122.74 & C-C-C Angle & $\mathrm{mPW} 1 \mathrm{PW} 91^{b}$ \\
\hline 122.01 & 114.52 & C-C-O Angle & mPW1PW91 $^{b}$ \\
\hline 128.16 & 128.86 & C-C-O Angle & mPW1PW91 $^{b}$ \\
\hline 109.82 & 116.62 & O-C-O Angle & mPW1PW91 $^{b}$ \\
\hline 110.33 & 102.87 & C-O-H Angle & mPW1PW91 ${ }^{b}$ \\
\hline
\end{tabular}


Table S3. Continued

\begin{tabular}{|c|c|c|c|}
\hline $\begin{array}{l}\text { Ala } \\
\text { SRP-AM1 }\end{array}$ & Exp/ab-initio & Prop. & Source \\
\hline-100.84 & -99.12 & Heat of formation & $\operatorname{Exp}^{c}$ \\
\hline 371.65 & 370.0 & $\begin{array}{l}\text { Proton Affinity } \\
\text { Heat of formation }\end{array}$ & mPW1PW91 $1^{b}$ \\
\hline-42.67 & ND & $\begin{array}{l}\text { Zwitter-ion } \\
\text { Proton Affinity }\end{array}$ & \\
\hline 381.98 & 381.29 & Zwitter-ion & \\
\hline 1.4487 & 1.4660 & C-N Bond & mPW1PW91 ${ }^{b}$ \\
\hline 1.1208 & 1.0961 & C-H Bond & mPW1PW91 ${ }^{b}$ \\
\hline 1.5299 & 1.5260 & C-C Bond & mPW1PW91 ${ }^{b}$ \\
\hline 0.9689 & 0.9888 & O-H Bond & mPW1PW91 ${ }^{b}$ \\
\hline 0.9948 & 1.0144 & N-H Bond & mPW1PW91 ${ }^{b}$ \\
\hline 0.9949 & 1.0139 & N-H Bond & mPW1PW91 \\
\hline 1.5372 & 1.5377 & C-C Bond & mPW1PW91 ${ }^{b}$ \\
\hline 1.1089 & 1.0952 & C-H Bond & mPW1PW91 ${ }^{b}$ \\
\hline 1.1100 & 1.0915 & C-H Bond & mPW1PW91 ${ }^{b}$ \\
\hline 1.1085 & 1.0948 & C-H Bond & mPW1PW91 ${ }^{b}$ \\
\hline 1.3406 & 1.3334 & C-O Bond & mPW1PW91 \\
\hline 1.2175 & 1.2062 & C-O Bond & mPW1PW91 \\
\hline 107.03 & 112.32 & N-C-C Angle & mPW1PW91 \\
\hline 113.60 & 111.04 & N-C-C Angle & mPW1PW91 ${ }^{b}$ \\
\hline 113.24 & 109.15 & C-C-C Angle & mPW1PW91 ${ }^{b}$ \\
\hline 107.11 & 109.02 & C-C-H Angle & mPW1PW91 \\
\hline 123.11 & 113.99 & C-C-O Angle & mPW1PW91 ${ }^{b}$ \\
\hline 122.72 & 122.61 & C-C-O Angle & mPW1PW91 \\
\hline 114.13 & 123.39 & O-C-O Angle & mPW1PW91 \\
\hline 113.46 & 104.85 & $\mathrm{C}-\mathrm{O}-\mathrm{H}$ Angle & mPW1PW91 ${ }^{b}$ \\
\hline 5.6709 & 5.5159 & Dipole moment & mPW1PW91 ${ }^{b}$ \\
\hline 3598.0 & 3646.4 & Frequency & mPW1PW91 ${ }^{b}$ \\
\hline 3530.9 & 3556.4 & Frequency & mPW1PW91 ${ }^{b}$ \\
\hline 3505.7 & 3421.1 & Frequency & mPW1PW91 ${ }^{b}$ \\
\hline 3232.5 & 3187.4 & Frequency & mPW1PW91 ${ }^{b}$ \\
\hline 3144.4 & 3150.7 & Frequency & mPW1PW91 ${ }^{b}$ \\
\hline 3138.8 & 3087.5 & Frequency & mPW1PW91 ${ }^{b}$ \\
\hline 3068.5 & 3076.6 & Frequency & mPW1PW91 ${ }^{b}$ \\
\hline
\end{tabular}

${ }^{a}$ Energies are in units of $\mathrm{kcal} / \mathrm{mol}$, distances in $\AA$, angles in degrees, dipole moments in Debye, atomic charges in a.u., and frequencies in $\mathrm{cm}^{-1} .{ }^{b} \mathrm{mPW} 1 \mathrm{PW} 91 / 6-$

$311++\mathrm{G}(3 \mathrm{df}, 2 \mathrm{p}) / / \mathrm{mPW} 1 \mathrm{PW} 91 / 6-31+\mathrm{G}(\mathrm{d}){ }^{c}$ Hunter, E.P.; Lias, S.G. J. Phys. Chem. Ref.

Data 1998, 27, 413-656. 
Table S4. Average Distances for the Reactant, Transition, and Product States in the AlaRM Catalyzed Reaction. The averages were obtained from ca. 5000 frames from a MD trajectory spanning 50ps.

\begin{tabular}{|c|c|c|c|c|c|c|c|c|c|c|c|c|}
\hline \multirow[b]{2}{*}{$\operatorname{Arg} 136$} & \multirow[b]{2}{*}{$\mathrm{NE}$} & \multirow[b]{2}{*}{ - Kcx129 } & \multirow[b]{2}{*}{ OX2 } & \multicolumn{3}{|c|}{ Reactant state } & \multicolumn{3}{|c|}{ Transition state } & \multicolumn{3}{|c|}{ Product state } \\
\hline & & & & 2.73 & \pm & 0.09 & 2.79 & \pm & 0.15 & 3.15 & \pm & 0.83 \\
\hline Arg136 & $\mathrm{NH} 2$ & $-\quad$ Kcx129 & OX1 & 2.68 & \pm & 0.09 & 2.64 & \pm & 0.08 & 2.69 & \pm & 0.13 \\
\hline Arg136 & NH1 & - W102 & $\mathrm{OH} 2$ & 3.30 & \pm & 0.29 & 2.99 & \pm & 0.18 & 3.40 & \pm & 0.26 \\
\hline Gln314' & $\mathrm{N}$ & - W102 & $\mathrm{OH} 2$ & 2.97 & \pm & 0.33 & 2.89 & \pm & 0.13 & 2.83 & \pm & 0.10 \\
\hline His 166 & ND1 & - $\quad \operatorname{Arg} 219$ & NH1 & 2.92 & \pm & 0.11 & 3.04 & \pm & 0.14 & 3.03 & \pm & 0.15 \\
\hline Lys39 & $\mathrm{NZ}$ & - Tyr43 & $\mathrm{OH}$ & 2.95 & \pm & 0.17 & 2.95 & \pm & 0.17 & 3.08 & \pm & 0.34 \\
\hline Lys39 & $\mathrm{NZ}$ & - Asp313' & OD2 & 2.65 & \pm & 0.08 & 2.67 & \pm & 0.08 & 2.69 & \pm & 0.09 \\
\hline Tyr265' & $\mathrm{OH}$ & - PLP & $\mathrm{CA}$ & 3.20 & \pm & 0.11 & 2.58 & \pm & 0.05 & 3.24 & \pm & 0.11 \\
\hline Tyr265, & $\mathrm{OH}$ & - PLP & HA & 2.16 & \pm & 0.08 & 1.20 & \pm & 0.04 & 0.99 & \pm & 0.02 \\
\hline PLP & $\mathrm{CA}$ & - PLP & HA & 1.14 & \pm & 0.02 & 1.43 & \pm & 0.04 & 2.40 & \pm & 0.08 \\
\hline PLP & N1 & - $\quad \operatorname{Arg} 219$ & $\mathrm{NE}$ & 2.56 & \pm & 0.08 & 2.53 & \pm & 0.07 & 2.52 & \pm & 0.07 \\
\hline PLP & $\mathrm{O} 3 \mathrm{~A}$ & - $\quad$ Arg136 & NH1 & 2.94 & \pm & 0.24 & 2.84 & \pm & 0.16 & 2.73 & \pm & 0.15 \\
\hline PLP & $\mathrm{O} 3 \mathrm{~A}$ & - $\quad$ Arg136 & $\mathrm{NH} 2$ & 2.74 & \pm & 0.11 & 2.64 & \pm & 0.10 & 2.71 & \pm & 0.19 \\
\hline PLP & $\mathrm{O}$ & - Met312 & $\mathrm{N}$ & 2.81 & \pm & 0.13 & 2.67 & \pm & 0.11 & 2.83 & \pm & 0.14 \\
\hline PLP & OXT & - $\operatorname{Arg} 136$ & NH1 & 2.71 & \pm & 0.13 & 3.10 & \pm & 0.27 & 2.64 & \pm & 0.11 \\
\hline PLP & OXT & - W102 & $\mathrm{OH} 2$ & 2.63 & \pm & 0.11 & 2.50 & \pm & 0.09 & 2.48 & \pm & 0.10 \\
\hline PLP & OP2 & - W112 & $\mathrm{OH} 2$ & 2.62 & \pm & 0.10 & 2.78 & \pm & 0.24 & 2.65 & \pm & 0.11 \\
\hline PLP & OP2 & - Asn203 & ND2 & 3.22 & \pm & 0.24 & 3.15 & \pm & 0.21 & 3.03 & \pm & 0.19 \\
\hline PLP & OP2 & - $\quad$ Tyr354 & $\mathrm{OH}$ & 2.64 & \pm & 0.11 & 2.59 & \pm & 0.09 & 2.57 & \pm & 0.08 \\
\hline PLP & OP1 & - Ser204 & OG & 2.60 & \pm & 0.09 & 2.59 & \pm & 0.08 & 2.60 & \pm & 0.08 \\
\hline PLP & OP1 & - Ser204 & $\mathrm{N}$ & 3.11 & \pm & 0.25 & 3.29 & \pm & 0.34 & 3.06 & \pm & 0.19 \\
\hline PLP & OP3 & - Tyr43 & $\mathrm{OH}$ & 2.56 & \pm & 0.08 & 2.57 & \pm & 0.08 & 2.58 & \pm & 0.08 \\
\hline PLP & OP3 & - $\quad$ Ile222 & $\mathrm{N}$ & 2.95 & \pm & 0.21 & 2.99 & \pm & 0.30 & 2.84 & \pm & 0.14 \\
\hline Tyr265' & $\mathrm{OH}$ & - His166 & NE2 & 2.88 & \pm & 0.11 & 2.88 & \pm & 0.10 & 3.13 & \pm & 0.20 \\
\hline Tyr265' & $\mathrm{OH}$ & - W112 & $\mathrm{OH} 2$ & 2.90 & \pm & 0.15 & 2.96 & \pm & 0.17 & 3.25 & \pm & 0.24 \\
\hline
\end{tabular}

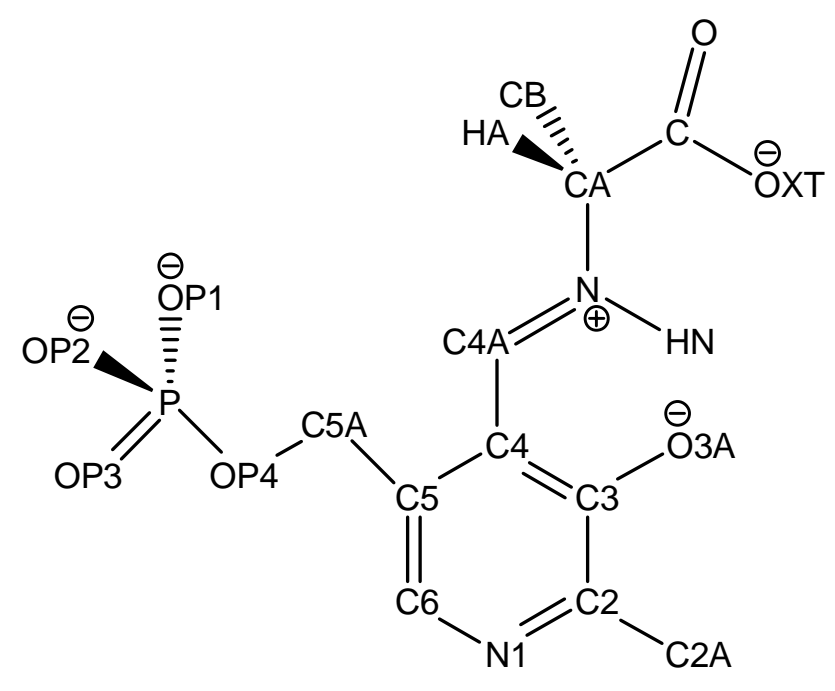

